\title{
mito-TEMPO Attenuates Oxidative Stress and Mitochondrial Dysfunction in Noise-Induced Hearing Loss via Maintaining TFAM-mtDNA Interaction and Mitochondrial Biogenesis
}

\author{
Jia-Wei Chen ${ }^{1 \dagger}$, Peng-Wei Ma ${ }^{1 \dagger}$, Hao Yuan ${ }^{1 \dagger}$, Wei-Long Wang ${ }^{1}$, Pei-Heng Lu ${ }^{1}$, \\ Xue-Rui Ding ${ }^{1}$, Yu-Qiang Lun ${ }^{1}$, Qian Yang ${ }^{2 *}$ and Lian-Jun Lu ${ }^{1 *}$ \\ 'Department of Otolaryngology Head and Neck Surgery, Tangdu Hospital, Fourth Military Medical University, Xi'an, China, \\ ${ }^{2}$ Department of Experimental Surgery, Tangdu Hospital, Fourth Military Medical University, Xi'an, China
}

OPEN ACCESS

Edited by:

Zuhong He,

Wuhan University, China

Reviewed by:

Zheng-De Du,

Capital Medical University, China Hangkang Chen,

Tri-Service General Hospital, Taiwan

*Correspondence:

Lian-Jun Lu

Iulianj@fmmu.edu.cn

Qian Yang

qianyang@fmmu.edu.cn

${ }^{\dagger}$ These authors have contributed equally to this work

Specialty section: This article was submitted to Cellular Neuropathology, a section of the journal Frontiers in Cellular Neuroscience

Received: 28 October 2021 Accepted: 14 January 2022 Published: 08 February 2022

Citation:

Chen J-W, Ma P-W, Yuan H, Wang $W-L$, Lu $P-H$, Ding $X-R$, Lun $Y-Q$, Yang $Q$ and $L u L-J$ (2022) mito-TEMPO Attenuates Oxidative Stress and Mitochondrial

Dysfunction in Noise-Induced

Hearing Loss via Maintaining TFAM-mtDNA Interaction and

Mitochondrial Biogenesis.

Front. Cell. Neurosci. 16:803718. doi: 10.3389/fncel.2022.803718
The excessive generation of reactive oxygen species (ROS) and mitochondrial damage have been widely reported in noise-induced hearing loss (NIHL). However, the specific mechanism of noise-induced mitochondrial damage remains largely unclear. In this study, we showed that acoustic trauma caused oxidative damage to mitochondrial DNA (mtDNA), leading to the reduction of mtDNA content, mitochondrial gene expression and ATP level in rat cochleae. The expression level and mtDNA-binding function of mitochondrial transcription factor A (TFAM) were impaired following acoustic trauma without affecting the upstream PGC-1 $\alpha$ and NRF-1. The mitochondria-target antioxidant mito-TEMPO (MT) was demonstrated to enter the inner ear after the systemic administration. MT treatment significantly alleviated noise-induced auditory threshold shifts $3 d$ and $14 d$ after noise exposure. Furthermore, MT significantly reduced outer hair cell $(\mathrm{OHC})$ loss, cochlear ribbon synapse loss, and auditory nerve fiber (ANF) degeneration after the noise exposure. In addition, we found that MT treatment effectively attenuated noise-induced cochlear oxidative stress and mtDNA damage, as indicated by DHE, 4-HNE, and 8-OHdG. MT treatment also improved mitochondrial biogenesis, ATP generation, and TFAM-mtDNA interaction in the cochlea. These findings suggest that MT has protective effects against NIHL via maintaining TFAM-mtDNA interaction and mitochondrial biogenesis based on its ROS scavenging capacity.

Keywords: noise-induced hearing loss, reactive oxygen species, mitochondrial transcription factor A, mitochondrial biogenesis, sensory hair cells, rat model

\section{INTRODUCTION}

Hearing loss is the most prevalent sensory disorder and affects more than 1.5 billion people worldwide (WHO, 2021). Common causes of acquired sensorineural hearing loss include exposure to loud noise (Sha and Schacht, 2017), aminoglycosides (He et al., 2017), platinum-based chemotherapy (Fernandez et al., 2020), heavy metals (Ding et al., 2019), and aging (He et al., 2021a). With the rapid development of modern society, noise has become one of the most common environmental pollutants in industrial and recreational settings. Exposure to high-intensity or chronic noise leads to noise-induced hearing loss (NIHL). 
Moderate noise leads to temporary threshold shift (TTS) that completely recovers to normal in 1-2 weeks. However, high-intensity or chronic noise can result in permanent threshold shift (PTS), i.e., irreversible hearing loss (Kurabi et al., 2017). According to WHO, about $10 \%$ of the world's population is exposed to noise, 5.3\% of whom develop NIHL (WHO, 2021). Those who experience hearing loss have difficulties in social communication, which may cause potential mental and cognitive disorders such as anxiety, depression, and dementia (Loughrey et al., 2018; Blazer and Tucci, 2019).

The cochlea is a metabolically active auditory sensory organ. Loud noise causes a dramatic increase in energy consumption of the cochlea (Chen et al., 2012). Production of reactive oxygen species (ROS) was observed in the cochlea in a few minutes following noise exposure, including superoxide $\left(\mathrm{O}_{2} \cdot-\right)$, hydroxyl radical $(\cdot \mathrm{OH})$, and hydrogen peroxide $\left(\mathrm{H}_{2} \mathrm{O}_{2}\right)$. Moreover, ROS can be generated continuously for 7-10 days after cessation of the noise exposure (Ohlemiller et al., 1999; Yamashita et al., 2004). A lower level of ROS induced by moderate noise is reported to induce autophagy which protects against NIHL (Yuan et al., 2015). However, high-intensity noise induces excess ROS and activates 5 ' adenosine monophosphate-activated protein kinase (AMPK), leading to hair cell death and hearing loss ( $\mathrm{Wu}$ et al., 2020). Oxidative stress has been considered as a key factor in the mechanism of NIHL (Fetoni et al., 2019). ROS in the cochlea mainly comes from the mitochondrial metabolism of various cells. Mitochondrial pathology and dysfunction are associated with hearing loss of various etiologies (FischelGhodsian et al., 2004; Böttger and Schacht, 2013). Mitochondria are essential organelles with several vital functions including energy production, maintenance of calcium homeostasis, and regulation of apoptosis ( $\mathrm{Lin}$ and Beal, 2006). Due to the proximity to ROS production, mitochondria are also the first organelles damaged by ROS. The phenomenon of mitochondrial damage has been reported to be involved in NIHL in previous studies (Coleman et al., 2007; Park et al., 2012). However, the underlying mechanism has not been fully elucidated yet.

The mitochondrial DNA (mtDNA) is a circular doublestranded DNA located in the matrix of mitochondria, encoding 13 essential subunits of the mitochondrial respiratory chain (MRC; Scarpulla, 2008). Due to the lack of the repair mechanism and histone proteins like genomic DNA, mtDNA is more susceptible to the damage by ROS (Tsutsui et al., 2009). Mitochondrial transcription factor A (TFAM), a nucleus-encoded protein, is essential for the stabilization and maintenance of mtDNA. TFAM can bind to mtDNA in a sequence-independent manner to compact and stabilize the genome. In addition, TFAM can also bind sequencespecifically to promoter regions of mtDNA, initiating transcription and replication of mtDNA (Kang et al., 2007; Chandrasekaran et al., 2015). Previous studies have reported mitochondrial disintegration and vacuolization in hair cells (HCs) after acoustic trauma (Coleman et al., 2007; Park et al., 2012). However, the specific mechanism of noiseinduced mitochondrial damage remains unclear. There are few studies on the alterations of the cochlear mtDNA content and expression level after acoustic trauma. Whether TFAM dysregulation is involved in the pathogenesis of NIHL is still unknown.

Recently, several novel antioxidants that specifically target mitochondria have been developed for the treatment of oxidative stress-related diseases (Zinovkin and Zamyatnin, 2019). 2(2,2,6,6-tetramethylpiperidin-1-oxyl-4-ylamino)-2-oxoethyl

(mito-TEMPO) is a mitochondria-target antioxidant with strong superoxide scavenging capacity and several hundred-fold accumulations in mitochondria (Shetty et al., 2019). A previous study demonstrated that mito-TEMPO (MT) can ameliorate renal fibrosis by reducing oxidative stress, mitochondrial dysfunction, and inflammation (Liu et al., 2018). In addition, recent studies have shown that MT has a protective effect against acetaminophen-induced hepatotoxicity with a wider therapeutic time window than N-acetyl-L-cysteine (NAC; Du et al., 2017; Abdullah-Al-Shoeb et al., 2020). Systemic administration of MT was able to alleviate ischemic brain damage in rats ( $\mathrm{Li}$ et al., 2018). In addition, MT can pass through the blood-brain barrier (BBB) which is structurally similar to the blood-labyrinth barrier (BLB) in stria vascularis of the cochlea (Zhelev et al., 2013; Nyberg et al., 2019). However, whether MT has a protective effect on hearing has not been reported yet.

In the present study, we explored whether MT has a protective effect against NIHL and the underlying mechanism using an in vivo rat model. We first detected whether MT entered the inner ear after the systemic administration. Then we investigated the alleviation of noise-induced hearing loss, outer hair cell (OHC) loss, inner hair cell (IHC) ribbon synapse loss, and auditory nerve fiber (ANF) degeneration with systemic use of MT. We hypothesized that MT exerted the protective effect on hearing via reducing mtDNA oxidative damage, stabilizing the TFAM-mtDNA interaction, maintaining mitochondrial biogenesis and function.

\section{MATERIALS AND METHODS}

\section{Animals}

Sixty-two female adult Sprague-Dawley rats (200-250 g) were used in this study. Two rats were used for the determination of MT in the cochlea. Sixty Rats were randomly assigned to three groups ( $n=20$ for each group): control, noise + vehicle treatment, noise $+\mathrm{MT}$ treatment. All animals were kept at $22 \pm 1^{\circ} \mathrm{C}$ under a $12 \mathrm{~h} \mathrm{light} / 12 \mathrm{~h}$ dark cycle with free access to water and food. All procedures of the animal experiments were approved by the Institutional Animal Care and Use Committee of Fourth Military Medical University.

\section{Auditory Brainstem Response (ABR) Measurements}

The hearing function of 18 animals ( $n=6$ for each group) was evaluated by auditory brainstem response $(A B R)$ at four frequencies of $8,16,24$, and $32 \mathrm{kHz}$. ABR measurements were carried out 2 days before $(-2 \mathrm{~d}), 3$ days after $(+3 \mathrm{~d})$ and 14 days after $(+14 \mathrm{~d})$ the noise exposure to evaluate the baseline hearing function and noise-induced auditory threshold shifts. Rats were anesthetized with an intraperitoneal injection of the 
cocktail anesthetic (chloral hydrate, pentobarbital sodium, and magnesium sulfate). Body temperature was maintained at near $37^{\circ} \mathrm{C}$ with a heating pad. Three subdermal electrodes were inserted at the vertex of the skull (active), the left mastoid (reference), and the right mastoid (ground). Tucker-Davis Technologies (TDT) RZ6 System was used to generate acoustic stimuli and record the responses. Tone pip stimuli at 8, 16, 24, and $32 \mathrm{kHz}$ (5-ms-duration with 2.5-ms rise-fall time) were delivered monaurally with an earphone inserted into the external auditory canal of the left ear. Acoustic stimuli were generated from $90 \mathrm{~dB}$ and then attenuated in $10-\mathrm{dB}$ and $5-\mathrm{dB}$ steps until no $A B R$ waves were recognizable. A total of 1,024 responses were averaged for each stimulus level. ABR wave II was used to determine ABR thresholds for each frequency. The thresholds were defined as the lowest intensity able to evoke repeatable response waves.

\section{Drug Administration}

MT (Sigma-Aldrich, SML0737) was dissolved in normal saline as a stock solution and stored at $-20^{\circ} \mathrm{C}$. The stock solution was diluted with normal saline at a concentration of $0.5 \mathrm{mg} / \mathrm{ml}$ before the injection. For animals in the MT treatment group, a dose of $1 \mathrm{mg} / \mathrm{kg}$ was selected based on the previous studies (Liu et al., 2018; Xing et al., 2021). For the animals to be sacrificed on day 1, MT was injected intraperitoneally (i.p.) $24 \mathrm{~h}$ before, $1 \mathrm{~h}$ before, and immediately after noise exposure. The Animals to be sacrificed on day 3 and 14 received three additional injections once daily for the following 3 days. The animals in the control group and the vehicle treatment group received three or six injections of normal saline with the same experimental procedure.

\section{Perilymph Extraction and Sample Preparation}

Two rats were injected intraperitoneally with $1 \mathrm{mg} / \mathrm{kg}$ MT. Fifteen minutes after the injection, rats were sacrificed and the cochleae were harvested. Then $2 \mu \mathrm{l}$ of perilymph was sampled using a capillary tube from the round window of the cochlea. A total of $8 \mu \mathrm{l}$ of perilymph was pooled and used for the determination of MT. Then $100 \mu \mathrm{l}$ of methanol was added to the sample. After vortexing, the sample was centrifuged at $5,000 \times \mathrm{g}$ for $10 \mathrm{~min}$ at $4^{\circ} \mathrm{C}$. The supernatant was retained and used for liquid chromatography tandem mass spectrometry (LC-MS/MS) analysis.

\section{Chromatographic Separation and MS/MS Detection}

The LC-MS/MS analysis was performed with the Triple Quadrupole LC/MS (Agilent, 6470B, USA). Chromatographic separation was performed using an Agilent C18 column $(100 \times 2.1 \mathrm{~mm}$ i.d., $3 \mu \mathrm{m}$ particle size $)$. The sample injection volume was $10 \mu \mathrm{l}$. The column temperature was maintained at $35^{\circ} \mathrm{C}$. Then a gradient of mobile phase A $(0.1 \%(\mathrm{v} / \mathrm{v})$ formic acid in $5 \mathrm{mM}$ ammonium acetate) and mobile phase B (acetonitrile) was run at $0.3 \mathrm{ml} / \mathrm{min}$ as follows: $95 \% \mathrm{~A}$ and $5 \% \mathrm{~B}, 0-15 \mathrm{~min}$; $20 \% \mathrm{~A}$ and $80 \% \mathrm{~B}, 15-17 \mathrm{~min} ; 100 \% \mathrm{~A}, 17-20 \mathrm{~min}$. The MS/MS analysis was performed in the positive electrospray ionization
(ESI+) mode. The source temperature was kept at $500^{\circ} \mathrm{C}$. The ion spray voltage was $4,500 \mathrm{~V}$, and the curtain gas was set to 35 psi. The detection of the ions was operated in the multiple reaction monitoring (MRM) mode, monitoring transitions of $\mathrm{m} / \mathrm{z} 474.2$ $\rightarrow 320.1$ for MT. The quantification of the MT in the perilymph was analyzed by calculating the peak area ratios using the external standard.

\section{Acoustic Trauma}

The acoustic trauma was induced by continuous noise exposure. The rats were placed separately in wire cages located in a soundproof room. The acoustic signal of $8-16 \mathrm{kHz}$ octave band noise was generated by RZ6 Noise software. Then the signal was amplified and output by the power amplifier (Crown, XLi800, China) and four loudspeakers (CHUANGMU, CP-75A, China). The level of the noise was maintained at $112 \pm 1 \mathrm{~dB}$ SPL confirmed by the Sound Level Meter. Animals were exposed to noise for $2 \mathrm{~h}$.

\section{Immunofluorescence Histochemistry for Surface Preparations and Frozen Sections of the Cochlea}

Twelve animals used for the analyses of 4-HNE, DHE, 8-OHdG level, and TFAM-mtDNA interaction were sacrificed $1 \mathrm{~h}$ after the noise exposure. Eighteen animals used for analyses of OHC loss, IHC ribbon synapse loss, and ANF degeneration were sacrificed $14 \mathrm{~d}$ after the noise exposure. Rat cochleae were quickly dissected from the temporal bones following rapid decapitation. The round and oval windows were opened after removing the stapes. Then the cochleae were gently perfused with $4 \%$ paraformaldehyde (PFA) in $0.1 \mathrm{M}$ phosphate-buffered saline (PBS) via the round window, and kept in the fixative overnight at $4^{\circ} \mathrm{C}$. After the fixation, the cochleae were rinsed three times with PBS for $5 \mathrm{~min}$ and then decalcified with $10 \%$ ethylenediaminetetraacetic acid (EDTA) for 5 days at room temperature with a daily solution change. After the decalcification, the cochleae were rinsed three times with PBS for 5 min. For cochlear surface preparations, the organ of Corti (OC) was dissected in PBS by carefully removing the lateral wall, Reissner's membrane, and the tectorial membrane of the cochlea using micro-dissecting forceps and scissors under a stereomicroscope (Olympus, SZ61, Japan). Then the specimens were transferred into a 96-well plate, followed by permeabilization using $1 \%$ Triton X-100 in PBS for $15 \mathrm{~min}$ at room temperature. The specimens were blocked with immunol staining blocking buffer (Leagene, IH0338) for $1 \mathrm{~h}$ at room temperature. Then the specimens were incubated overnight at $4^{\circ} \mathrm{C}$ with the following primary antibodies: Myosin VIIa (Proteus Biosciences, \#25-6790, 1:1,000), TFAM (Abcam, ab252432, 1:500), dsDNA (Abcam, ab27156, 1:500). After being rinsed with $\mathrm{PBS}$ three times, the specimens were incubated for $2 \mathrm{~h}$ at room temperature in darkness with following secondary antibodies: donkey anti-rabbit IgG Alexa Fluor 594 (Invitrogen, A21207, 1:200), donkey anti-mouse IgG Alexa Fluor 488 (Invitrogen, A21202, 1:200). After being rinsed with PBS three times, the specimens were incubated with Acti-Stain 670 Phalloidin (Cytoskeleton, \#PHDN1-A, 1:70) or 
DAPI (Roche, \#10236276001, $1 \mu \mathrm{g} / \mathrm{ml}$ ) for $30 \mathrm{~min}$ at room temperature.

For immunolabeling of IHC ribbon synapses, the specimens were permeabilized with $3 \%$ Triton X-100 in PBS for $30 \mathrm{~min}$ at room temperature. After blocking for $1 \mathrm{~h}$, the specimens were incubated overnight at $37^{\circ} \mathrm{C}$ with following primary antibodies: Myosin VIIa (Proteus Biosciences, \#25-6790, 1:1,000), CtBP2 (BD Bioscience, \#612044, 1:500), GluR2 (Alomone Labs, AGC-005, 1:1,000). After being rinsed with PBS three times, the specimens were incubated for $2 \mathrm{~h}$ at room temperature in darkness with following secondary antibodies: goat anti-rabbit IgG Alexa Fluor 568 (Invitrogen, A11036, 1:200), goat anti-mouse IgG1 Alexa Fluor 647 (Invitrogen, A21240, 1:200), goat anti-mouse IgG2a Alexa Fluor 488 (Invitrogen, A21131, 1:200). After the final rinse with PBS, each specimen was dissected into the apex, middle and base turns. Each cochlear turn was transferred to the slide and placed in the mounting medium (Vector, H-1000). Finally, the specimen was covered with a coverslip and the edge was sealed with transparent nail polish.

For the preparation of cochlear frozen sections, the decalcified cochleae were incubated in $10 \%$ sucrose and $20 \%$ sucrose for $4 \mathrm{~h}$ and $30 \%$ sucrose overnight at $4^{\circ} \mathrm{C}$ for dehydration. Then the specimens were embedded in an optimal cutting temperature (OCT) compound. The midmodiolar cryosections at a thickness of $8 \mu \mathrm{m}$ were prepared using a Cryostat Microtome (Leica, CM1860, Germany). The sections were permeabilized with $0.5 \%$ Triton X-100 in PBS for $15 \mathrm{~min}$ at room temperature, followed by blocking with immunol staining blocking buffer for $1 \mathrm{~h}$ at room temperature. Then the sections were incubated overnight at $4{ }^{\circ} \mathrm{C}$ with following primary antibodies: Myosin VIIa (Proteus Biosciences, \#25-6790, 1:1,000), Tuj1 (GeneTex, GTX631836, 1:200), 4-HNE (GeneTex, GTX17571, 1:50), 8-OHdG (Abcam, ab48508, 1:200), Tuj1(ABclonal, A17913, 1:500). After being rinsed with $\mathrm{PBS}$ three times, the specimens were incubated with secondary antibodies for $2 \mathrm{~h}$ at room temperature in darkness. For DHE staining, the permeabilized sections were incubated with $1 \mu \mathrm{M}$ DHE solution (Beyotime, S0063) for $30 \mathrm{~min}$ at $37^{\circ} \mathrm{C}$ in darkness. After being rinsed with PBS three times, a drop of mounting medium with DAPI (Vector, H-1200) was added onto the section. Finally, the section was covered with a coverslip and the edge was sealed with transparent nail polish. Images were obtained with the Confocal Laser Scanning Microscope (CLSM; Olympus, FV1000, Japan) under identical parameter settings in each experiment.

\section{Quantitative or Semi-quantitative Analyses of the Fluorescence Signals}

The quantitative or semi-quantitative analyses of the fluorescence signals were conducted with ImageJ software (version 1.53a, USA). For the OHC counting 14d after noise exposure, cochlear surface preparations labeled with Myosin VIIa and DAPI were used. The OHC counting was measured in apex, middle and base turns of surface preparations in $0.3-\mathrm{mm}$ segments. The absence of both the nucleus (DAPI) and the cell body (Myosin VIIa) was considered as the OHC loss. The mean OHC survival in each group was calculated with four specimens. The quantification of IHC ribbon synapses was analyzed in the $0.1-\mathrm{mm}$ segment of the surface preparation (containing about 10 IHCs), corresponding to frequencies of $16-20 \mathrm{kHz}$. The juxtaposition of CtBP2 and GluA2 was considered as the paired synapse. The total number of paired fluorescent spots was counted and divided by the number of IHC within the image. For the analysis of ANF density, cochlear frozen sections immunolabelled with Tuj1 and Myosin VIIa were used. The intensity of Tuj1 fluorescent signals in nerve fibers in the upper-base turn of the cochlea was analyzed and considered as ANF density. For the semi-quantitative analyses of 8-OHdG and 4-HNE expression, the intensity of the fluorescent signals in SGNs, HCs, or SV region in the upper-base turn of the cochlea was analyzed. Briefly, the images were converted into 8 -bit grayscale type. The intensity of the background signal was subtracted and the average grayscale intensity of SGNs, HCs or $\mathrm{SV}$ region was then measured. The relative grayscale values were calculated by normalizing the ratio to the control group. The quantification of TFAM-mtDNA interaction was analyzed in the $70-\mu \mathrm{m}$ segment at the base turn of the surface preparation. Those green fluorescent spots (dsDNA) in the cytoplasm that were not colocalized with red ones (TFAM) were considered as naked mtDNA. The total naked mtDNA spots in OHCs were counted and divided by the number of $\mathrm{OHC}$ within the image. All quantitative and semi-quantitative analyses were performed in four specimens for each group.

\section{Extraction of Cochlear DNA and RNA}

Six animals were sacrificed on day 1 after the third injection and six animals were sacrificed on day 3 after the ABR testing. The cochleae were dissected in Hanks' balanced salt solution on ice and frozen in liquid nitrogen until use. Twelve cochleae ( $n=4$ for each group) of the animals sacrificed on day 1 were used for the extraction of total RNA. Twelve cochleae $(n=4$ for each group) of the animals sacrificed on day 3 were used for genomic DNA (gDNA) extraction. The gDNA and total RNA were extracted using the Tissue DNA Extraction Kit (TIANGEN, DP304) and RNeasy Plus Mini Kit (QIAGEN, \#74134) according to the manufacturer's instructions, respectively. The purity and concentration of the DNA and RNA products were analyzed using the microplate spectrophotometer (BioTek, Epoch, USA). Then the isolated RNA was reverse transcribed to cDNA using PrimeScript RT Master Mix (TaKaRa, RR036A). The gDNA and cDNA products were stored at $-20^{\circ} \mathrm{C}$ until use.

\section{Quantification of mtDNA Copy Number and Mitochondrial Nd6 mRNA Expression}

The mtDNA copy number and mitochondrial Nd6 expression were quantified by real-time polymerase chain reaction (PCR) assay using Real-Time PCR Detection System (Bio-Rad, CFX96, USA). The real-time PCR reaction system was prepared using TB Green Premix Ex Taq II (TaKaRa, RR820A) according to the manufacturer's instructions. The nuclear gene $\beta$-actin was used as the internal control. For the quantification of mtDNA copy number, the gDNA was used for the template. The cycle threshold (Ct) values of $12 \mathrm{~S}$ rRNA (mtDNA) and $\beta$-actin (nDNA) were used to determine the 
relative mtDNA copy number. The following primers were designed: $\beta$-actin Forward: CTACCTCGCTGCAGGATCG, $\beta$ actin Reverse: GTCTACACCGCGGGAATACG, 12S rRNA Forward: AAGGAGAGGGCATCAAGCAC, and 12S rRNA Reverse: TATCACTGCTGAGTCCCGTG.

For the measurement of mitochondrial Nd6 mRNA expression, the cDNA was used as a template. The relative mRNA expression was estimated by the $\mathrm{Ct}$ values of $\mathrm{Nd} 6$ (mtDNA expression) and $\beta$-actin (nDNA expression). The following primers were designed: $\beta$-actin Forward: GGAGATTA CTGCCCTGGCTCCTA, $\beta$-actin Reverse: GACTCATCGTAC TCCTGCTTGCTG, Nd6 Forward: ACCCTCAAGTCTCCGG GTA, and Nd6 Reverse: GTCTAGGGTTGGCGTTGAAG. The relative levels of mtDNA copy number and mRNA expression in each group were analyzed using the $2^{-\Delta \Delta \mathrm{Ct}}$ method.

\section{ATP Level Measurement}

Twelve animals ( $n=4$ for each group) in each group were sacrificed for cochlear ATP level measurement $1 \mathrm{~h}$ after the noise exposure. Two cochleae of an animal were quickly harvested and pooled as one sample. ATP levels in the cochlea were measured based on spectrophotometry using ATP Detection Kit (Solarbio, BC0305) according to the manufacturer's instructions. The working principle of the Kit is briefly described as follows. The Glucose and ATP are catalyzed by hexokinase to produce glucose 6-phosphate, which is further catalytically dehydrogenated to produce NADPH. The NADPH shows a characteristic absorption peak at $340 \mathrm{~nm}$. The content of ATP is proportional to that of NADPH.

\section{Extraction of Cochlear Protein and Immunoblotting}

Twelve cochleae ( $n=4$ for each group) of six animals sacrificed on day 1 were homogenized in ice-cold RIPA lysis buffer (Beyotime, P0013B) containing protease inhibitor cocktail (Roche, \#04693159001, Switzerland) using the cryogenic grinder. The tissue homogenate was placed on ice for $30 \mathrm{~min}$, and then centrifuged at $12,000 \times g$ for $10 \mathrm{~min}$ at $4^{\circ} \mathrm{C}$. The supernatant was retained and the protein concentration was determined using the BCA Determination Kit. Then the supernatant added with loading buffer was boiled for $5 \mathrm{~min}$ and stored at $-20^{\circ} \mathrm{C}$.

The protein samples $(30 \mu \mathrm{g})$ were separated by $10-12 \%$ sodium dodecyl sulfate polyacrylamide gel electrophoresis (SDS-PAGE) electrophoresis and transferred to polyvinylidene fluoride (PVDF) membranes (Millipore, IPVH00010). After being blocked with $5 \%$ skimmed milk in TBS-T, the membranes were incubated for $1 \mathrm{~h}$ at room temperature on shaker and then overnight at $4^{\circ} \mathrm{C}$ with following primary antibodies: PGC$1 \alpha$ (Abcam, ab191838, 1:1,000), NRF-1 (GeneTex, GTX103179, 1:1,000), TFAM (Abcam, ab131607, 1:1,000), SOD2(GeneTex, GTX116093, 1:1,000), Bax (GeneTex, GTX109683, 1:1,000), ACTB (GeneTex, GTX109639, 1:2,000). The membranes were rinsed three times with TBS-T for $5 \mathrm{~min}$, followed by incubation with HRP-linked secondary antibody (CST, \#7074, 1:2,000) for $1 \mathrm{~h}$ at room temperature on a shaker. After being rinsed with TBS-T three times, the blots were visualized with chemiluminescent HRP substrate (Millipore, WBKLS0100) and scanned using the Chemiluminescence Imaging System (Fusion, Solo 6S, China). The band intensities were quantified using the Image J software (version 1.53a, USA) and normalized by using ACTB as the internal control.

\section{Statistics}

Statistical analyses were conducted using IBM SPSS software (version 25.0, USA) and GraphPad Prism software (version 8.0, USA). The group comparisons were performed using two-tailed, two-sample Student's $t$-test or one-way analysis of variance (ANOVA). When the difference was significant, the LSD post hoc test was used to identify the difference between the two groups. The $p$-value of $<0.05$ was considered statistical significance. All the data were presented as mean \pm standard deviation (SD).

\section{RESULTS}

\section{MT Entered Inner Ear After Systemic Administration}

In order to investigate whether MT existed in the inner ear after the systemic administration, we analyzed the concentration of MT in the perilymph of the cochlea using LC-MS/MS. The perilymph was extracted $15 \mathrm{~min}$ after the injection and used for the detection and quantification of MT. As shown in Supplementary Figures 1A-C, the retention time of the MT in the perilymph was $3.302 \mathrm{~min}$. The mass-to-charge ratio $(\mathrm{m} / \mathrm{z})$ of the parent ion was 474.2 , which was consistent with the relative molecular mass of MT losing a chloride ion (Supplementary Figure 1D). The concentration of the MT in the perilymph of the cochlea was $18.541 \mu \mathrm{g} / \mathrm{kg} 15 \mathrm{~min}$ after the injection. These results indicated that MT could pass through the BLB and enter the inner ear.

\section{Administration of MT Attenuated Noise-Induced Auditory Threshold Shift}

To address whether systemic use of MT might prevent NIHL, we designed the experimental procedure as shown in Figure 1. The hearing function of the animals was evaluated via ABR testing 2 days before $(-2 \mathrm{~d}), 3$ and 14 days after $(+3 \mathrm{~d}$ and $+14 \mathrm{~d})$ noise exposure. There is no significant difference in baseline ABR threshold between groups (Supplementary Figure 2). Compared with saline-treated rats, MT-treated rats showed significantly decreased TTS on day 3 and PTS on day 14 at 8 , 16,24 , and $32 \mathrm{kHz}$ (Figures $2 \mathrm{~A}-\mathbf{D}$ ). The $112 \mathrm{~dB}$ noise exposure for $2 \mathrm{~h}$ resulted in about $30-40 \mathrm{~dB}$ threshold shift at all four frequencies on day 14 in the vehicle-treated group. However, in the MT-treated group, the threshold at $8 \mathrm{kHz}$ was completely recovered and threshold shifts at 24 and $32 \mathrm{kHz}$ were both within $10 \mathrm{~dB}$ on day 14 (Figures 2E,F). These data indicated that MT administration was able to attenuate noise-induced transient and permanent hearing loss.

\section{MT Treatment Prevented OHC Loss Induced by Acoustic Trauma}

We then asked if MT exhibits a protective effect on the $\mathrm{OHC}$, which is one of the most vulnerable structures of the cochlea during acoustic trauma. We used the surface preparation 


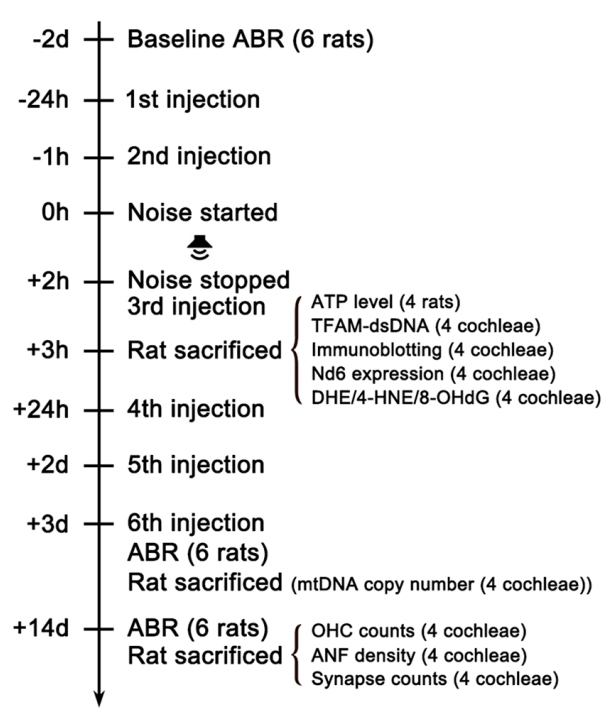

FIGURE 1 | Experimental timeline. The baseline hearing function of the rats was evaluated via ABR testing 2 days (2d) before the noise exposure. Rats were intraperitoneally injected with MT or vehicle (saline) $24 \mathrm{~h}$ and $1 \mathrm{~h}$ before the noise started. Then rats were exposed to $112 \mathrm{~dB}$ SPL 8-16 kHz octave band noise for $2 \mathrm{~h}$. The third shot was injected immediately after the noise stopped. One hour later, 12 animals in each group were sacrificed for analyses of oxidative stress level, mtDNA expression quantification, ATP measurement, immunoblotting and TFAM-mtDNA interaction. The rest of the animals received three additional injections once daily for the following 3 days. Then ABR testing were performed $3 d$ after the noise exposure to evaluate TTS level. Then two animals in each group were sacrificed for the quantification of cochlear mtDNA copy number. After ABR testing 14d after the noise exposure for PTS level assessment, the remaining animals were sacrificed for analyses of $\mathrm{OHC}$ loss, $\mathrm{IHC}$ ribbon synapse loss and ANF degeneration. ABR, Auditory Brainstem Response.

technique to analyze the $\mathrm{OHC}$ loss 14 days after the noise exposure. The cochlea was divided into the apex, middle and base turns, which correspond to the low-, middle- and high-frequency regions of the cochlea. The loss of both the nucleus (DAPI) and the cell body (Myosin VIIa) was considered as the OHC loss. As shown in Figures 3A,B, noise overstimulation resulted in the most $\mathrm{OHC}$ loss at the base turn in the vehicle-treated group, showing $81.5 \%$ of the $\mathrm{OHC}$ survival. Compared with the vehicletreated group, MT treatment significantly reduced noise-induced $\mathrm{OHC}$ loss at the base and middle turns of the cochlea. The OHC survival reached $95.2 \%$ at the base turn in the MT-treated group. These results indicated that MT was able to prevent $\mathrm{OHC}$ death after acoustic trauma.

\section{MT Treatment Reduced IHC Ribbon Synapse Loss and ANF Degeneration Induced by Acoustic Trauma}

Recent studies show that the loss of ribbon synapses between IHCs and spiral ganglion neurons (SGNs) is the primary pathology in NIHL, which is termed cochlear synaptopathy (Liberman and Kujawa, 2017). To explore the protective effect of MT against noise-induced IHC ribbon synapse loss, we quantified the number of paired ribbon synapses 14 days after the noise exposure. The surface preparations of the cochlea at approximately $16-20 \mathrm{kHz}$ region were immunolabelled with antibodies against CtBP2 and GluA2 to manifest the preand post-synaptic structures. The juxtaposition of CtBP2 and GluA2 represents the functional synapses. In the vehicle-treated group, a significant decrease in the number of paired synapses was observed after noise exposure. Compared with the vehicletreated group, treatment with MT significantly reduced the noise-induced loss of the paired ribbon synapses (Figures 4A,B).

In addition, NIHL is associated with the degeneration of ANFs which extend from SGNs to IHC ribbon synapses (Kujawa and Liberman, 2009). We next explored the protective effect of MT against noise-induced ANF degeneration by measuring the density of the fiber 14 days after the noise exposure. Compared with the control group, there was more than $50 \%$ degeneration of ANF in the vehicle-treated group. The remaining nerve fibers were fragmented and in a disordered arrangement. However, treatment with MT significantly recovered the density of ANF after acoustic trauma. The integrity of nerve fibers was partially restored in the MT-treated group (Figures 4C,D). These results indicated the neuroprotective effect of the MT against IHC ribbon synapse loss and ANF degeneration in NIHL.

\section{MT Treatment Mitigated Oxidative Stress in the Cochlea After the Noise Exposure}

Excessive ROS production is a major causative factor in noise-induced cochlear injury and hearing loss. ROS oxidizes polyunsaturated fatty acids to produce cytotoxic aldehydes. Among these, 4-hydroxynonenal (4-HNE) represents one of the major products of lipid peroxidation (Di Domenico et al., 2017). Dihydroethidium (DHE) is a fluorescent dye for detecting the level of superoxide ion $\left(\mathrm{O}_{2} \cdot-\right)$. In the presence of $\mathrm{O}_{2} \cdot-$, DHE is oxidized to 2-hydroxyethidium, the fluorescence of which can be measured at an excitation and emission wavelength of $480 \mathrm{~nm}$ and $567 \mathrm{~nm}$ (Fetoni et al., 2015). In the present study, the level of cochlear oxidative stress was analyzed by 4-HNE and DHE fluorescent staining. As shown in Figure 5A, noise overstimulation induced a substantial increase of 4-HNE generation in the cochlea. Increased 4-HNE was mainly observed in HCs, stria vascularis (SV), and SGNs of the cochlea (Supplementary Figure 3). Compared with the vehicle-treated group, MT treatment significantly attenuated 4-HNE generation after the noise exposure. The DHE assay showed similar results. MT treatment remarkably reduced the DHE fluorescence induced by the noise overstimulation (Figure 5B). These data indicated that MT alleviated noise-induced oxidative stress in the cochlea by reducing the level of lipid peroxidation and $\mathrm{O}_{2} \cdot-$ generation.

\section{MT Treatment Alleviated mtDNA Oxidative Damage, and Maintained Mitochondrial Biogenesis and ATP Production After the Acoustic Trauma}

The mitochondria are semi-autonomous organelles that contain their own circular genetic system. The mitochondrial genomes encode 13 essential protein subunits of the MRC complexes. 


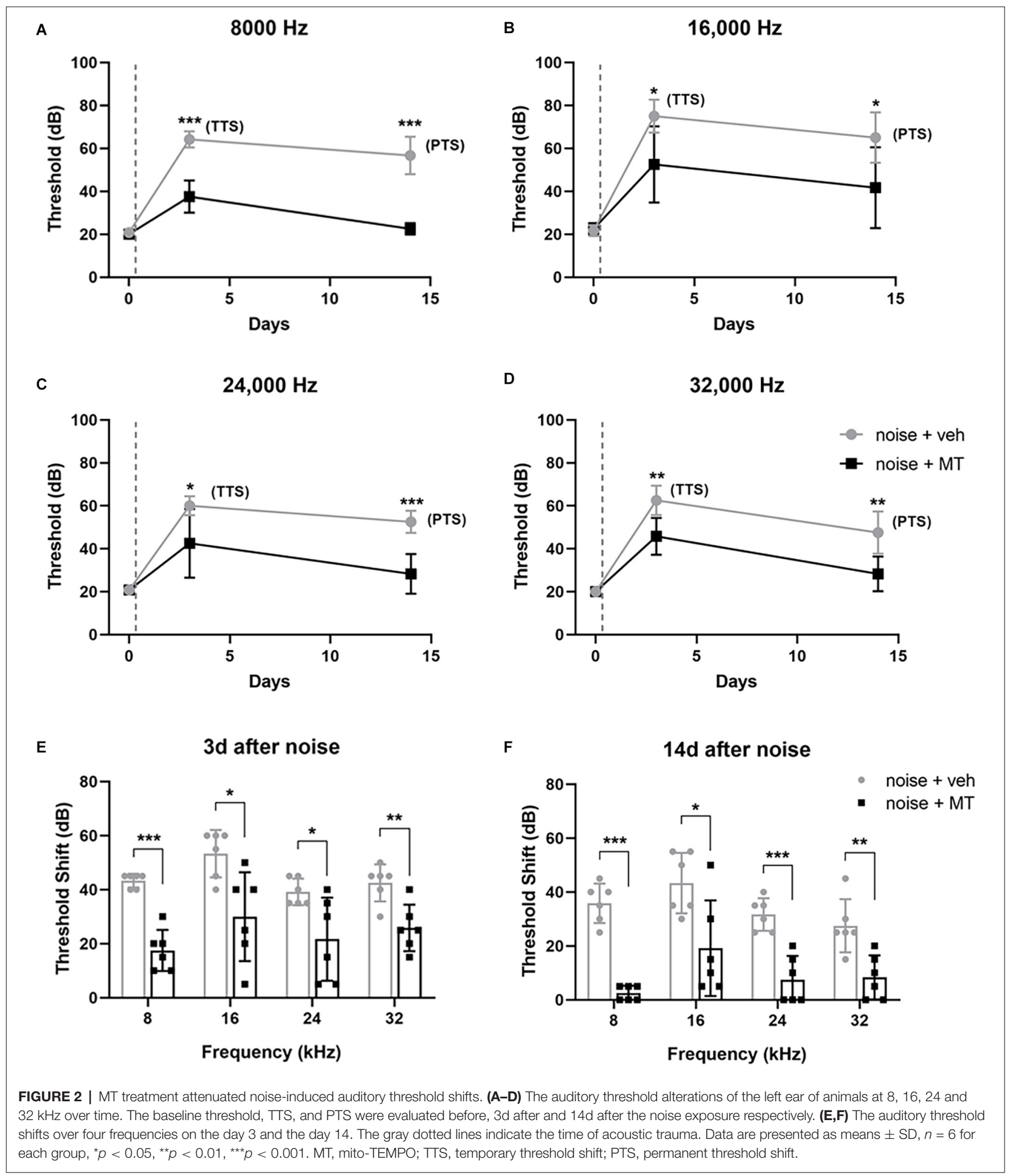

As is located close to the site of ROS generation, mtDNA molecules are easily damaged, resulting in decreased mtDNA content and mitochondrial dysfunction (Kang et al., 2007). 8 -hydroxy-2'-deoxyguanosine (8-OHdG) is one of the most abundant oxidative adducts of DNA, reflecting the level of DNA oxidative damage (Han et al., 2020). In Figures 6A,B, a dramatic increase of 8-OHdG production was observed in the cytoplasm of SGNs following noise exposure. Since the DNA in cytoplasm 

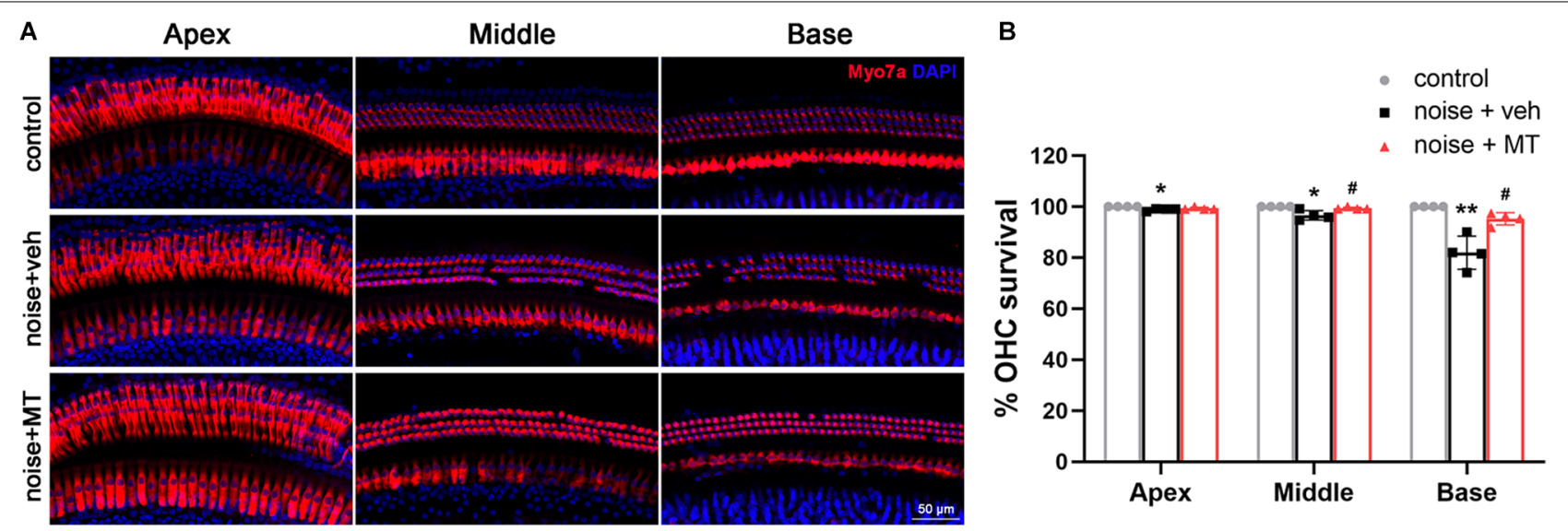

FIGURE 3 | MT treatment prevented noise-induced OHC loss. (A) Representative images of the apex, middle and base turn region of cochlear surface preparations 14 days after the noise exposure. HCs were indicated by Myosin VIla (red) and DAPI (blue) fluorescent staining. (B) Quantification analysis of OHC survival in the apex, middle and base turn region of the cochlea 14 days after the noise exposure. Data are presented as means \pm SD, $n=4$ for each group, ${ }^{\star} p<0.05$ vs. control group, ${ }^{* *} p<0.01$ vs. control group, ${ }^{\#} p<0.05$ vs. vehicle-treated group. OHC, outer hair cell; HCs, hair cells.

refers to mtDNA, increased cytoplasmic 8-OHdG indicated that acoustic trauma-induced mtDNA oxidative damage in SGNs. Compared with the vehicle-treated group, treatment of MT significantly reduced noise-induced 8 -OHdG production in SGNs.

Mitochondrial biogenesis relies on mtDNA replication and transcription to maintain mtDNA content and expression, and to produce new mitochondria. mtDNA is a multicopy genome and maintained at a relatively stable content (Scarpulla, 2008). Noise exposure resulted in a significant reduction of mtDNA content in the cochlea, reflected by the mtDNA copy number (Figure 6C). The mRNA level of MRC gene mt-Nd6 was also decreased after noise exposure, suggesting that the mtDNA expression in the cochlea was impaired by acoustic trauma (Figure 6D). In addition, ATP level in the cochlea was decreased following noise exposure, which indicated the dysfunction of the MRC (Figure 6E). However, treatment with MT significantly restored mtDNA content, mtDNA expression, and ATP level in the cochlea after noise exposure compared with the vehicle-treated group. These data indicated that MT was able to alleviate noiseinduced mtDNA oxidative damage, maintain mitochondrial biogenesis and improve mitochondrial function of the cochlea.

\section{MT Treatment Mitigated Noise-Induced Downregulation of TFAM and SOD2 Independent of the PGC-1 $\alpha /$ NRF-1/TFAM Pathway}

Mitochondrial biogenesis is a dynamic process of the generation of new mitochondria by synthesizing mitochondrial DNA and proteins. This activity requires several nucleus-encoded transcription factors, including peroxisome proliferatoractivated receptor-gamma coactivator-1alpha (PGC-1 $\alpha$ ), nuclear respiratory factor 1 and 2 (NRF-1 and NRF-2), mitochondrial transcription factor A (TFAM), B1 (TFB1M), and B2 (TFB2M; Scarpulla, 2008). The PGC-1 $\alpha /$ NRF-1/TFAM axis is the most studied pathway in regulating the replication and expression of
mtDNA and mitochondrial biogenesis (Zhao et al., 2013; Xiong et al., 2019). In the above experiment, we found that acoustic trauma led to the decline of mtDNA content and expression level. To further explore the underlying mechanism, we next investigated the expression of PGC- $1 \alpha / \mathrm{NRF}-1 / \mathrm{TFAM}$ after noise exposure. As shown in Figures 7A-D, the expression level of PGC- $1 \alpha$ and NRF-1 did not change following noise exposure. However, noise overstimulation resulted in a significant decrease in TFAM expression in the cochlea (Figures 7A,E). These results indicated that noise-induced decline of mtDNA content and expression was associated with TFAM reduction independent of PGC- $1 \alpha /$ NRF-1/TFAM pathway. Compared with the vehicletreated group, MT treatment attenuated TFAM expression reduction after the noise exposure.

Of the family of superoxide dismutase (SODs), only SOD2 (Mn-SOD) is located in mitochondria (Gross et al., 2014). We observed that noise exposure induced a dramatic decrease in the expression of mitochondrial SOD2 (Figures 7A,F). MT treatment partially improved SOD2 expression compared with the vehicle-treated group. In addition, the expression of the pro-apoptotic protein Bax was slightly upregulated following acoustic trauma and alleviated by MT treatment (Figures 7B,G). These data suggested that MT prevented noiseinduced disruption of the mitochondrial antioxidant defense, and inhibited apoptosis activation in the inner ear.

\section{MT Treatment Improved the Disruption of TFAM-mtDNA Interaction in the Cochlea After the Noise Exposure}

TFAM binds the D-loop region of the mitochondrial genome to initiate mtDNA transcription and replication. TFAM also maintains the stability of mtDNA in a sequence-independent binding manner. We investigated the mtDNA-binding ability of TFAM by immunofluorescence colocalization analysis of TFAM and the double-stranded DNA (dsDNA) in the cytoplasm. As shown in Figure 8A, most mtDNA molecules were bound 


\section{A}
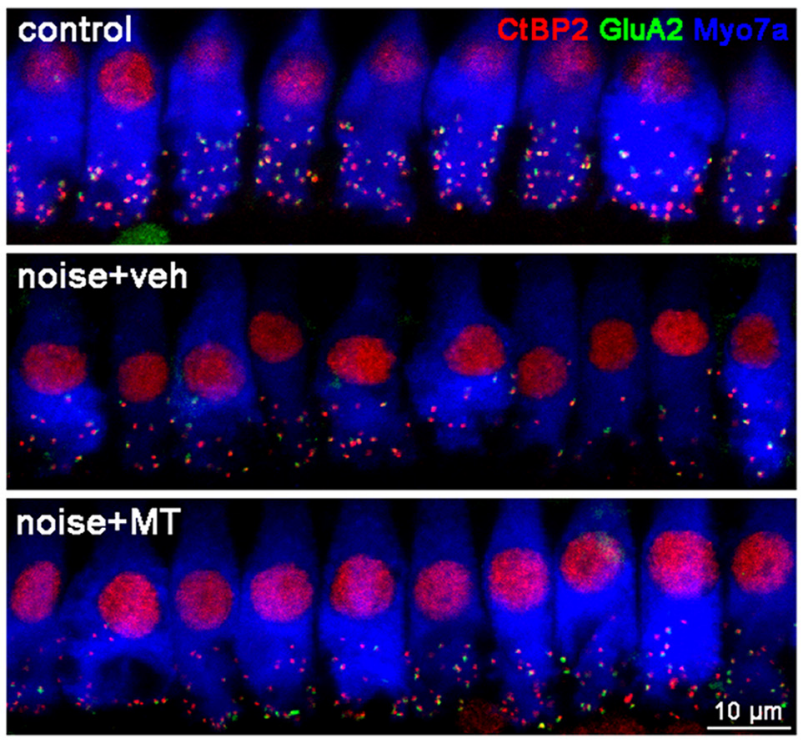

B

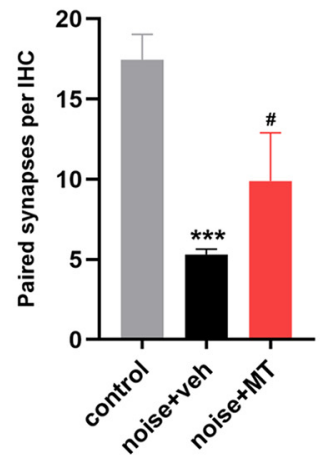

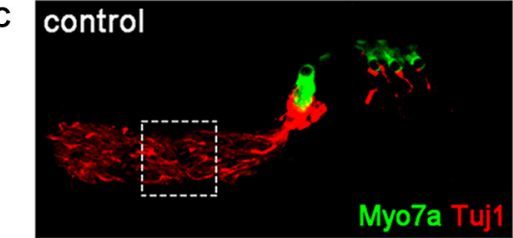
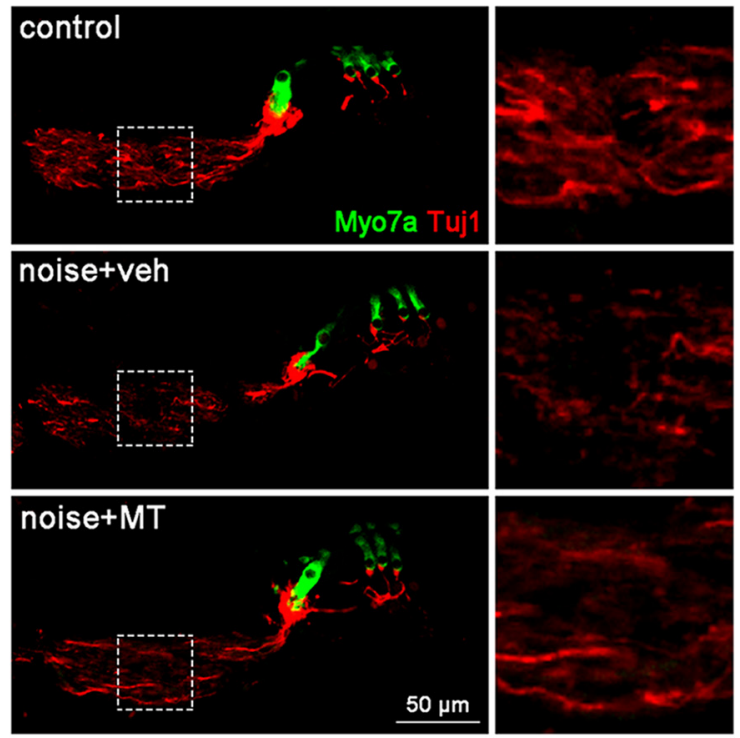

D

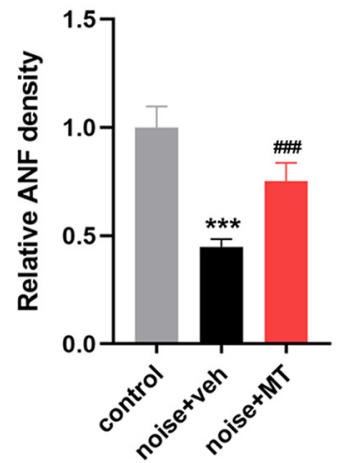

FIGURE 4 | MT treatment reduced IHC ribbon synapse loss and ANF degeneration induced by acoustic trauma. (A) Representative images of the IHC ribbon synapse at approximately 16-20 kHz region of the cochlea 14d after the noise exposure. Cochlear surface preparations were immunolabelled with CtBP2 (red), GluA2 (green) and Myosin VIla (blue). (B) Quantification analysis of paired synapse per IHC, indicated by the juxtaposition of CtBP2 and GluA2. (C) Representative images of ANF and HCs in middle turn area of the midmodiolar sections 14d after the noise exposure. Cochlear sections were immunolabelled with Tuj1 (red) and Myosin Vlla (green). (D) Quantification analysis of ANF density 14d after the noise exposure. Data are presented as means \pm SD, $n=4$ for each group, ${ }^{\star * \star} p<0.001$ vs. control group, ${ }^{\#} p<0.05$ vs. vehicle-treated group, ${ }^{\# \# \# ~} p<0.001$ vs. vehicle-treated group. $I H C$, inner hair cell; ANF, auditory nerve fiber.

to TFAM (yellow arrows) within sensory hair cells labeled by Phalloidin in the control group. However, noise exposure induced a reduction of TFAM-mtDNA interaction, leading to a significant increase of naked mtDNA (white arrows) in the cytoplasm of OHCs. MT treatment partially recovered the TFAM-mtDNA interaction and significantly alleviated the increase of naked mtDNA in OHCs (Figures 8A,B). These data indicated that the mtDNA-binding activity of TFAM was weakened in the cochlea following acoustic trauma. MT treatment could restore the binding function of TFAM.

\section{DISCUSSION}

MT is a novelly developed mitochondria-target antioxidant with the ability to pass through the phospholipid bilayer and accumulate in mitochondria. In the present study, we investigated the protective effect of MT against NIHL and the underlying mechanism in a rat model. We found that MT reduced noise-induced threshold shift and prevented OHC loss, IHC ribbon synapse loss, and ANF degeneration after the noise exposure. We demonstrated that MT exerted its protective effect on hearing by reducing the level of oxidative stress and mtDNA damage, restoring TFAM-mtDNA interaction and mitochondrial function of the inner ear.

HCs and SGNs are two important cells responsible for the perception, mechanoelectrical transduction, and transmission of auditory signals. We found that acoustic trauma led to $\mathrm{OHC}$ loss while IHC remained intact. This differential vulnerability may be due to the difference in intrinsic antioxidant capacity between IHCs and OHCs (Sha et al., 2001; Rosenhall et al., 2019). However, the amount of paired ribbon synapses between IHCs and SGNs was significantly reduced after the noise exposure. ANFs are the peripheral neurites of SGNs. Noise-induced ANF degeneration was observed in our experiments. In addition, the damage of cochlear ribbon synapses and ANFs is also the pathologic change of noise-induced hidden hearing loss 

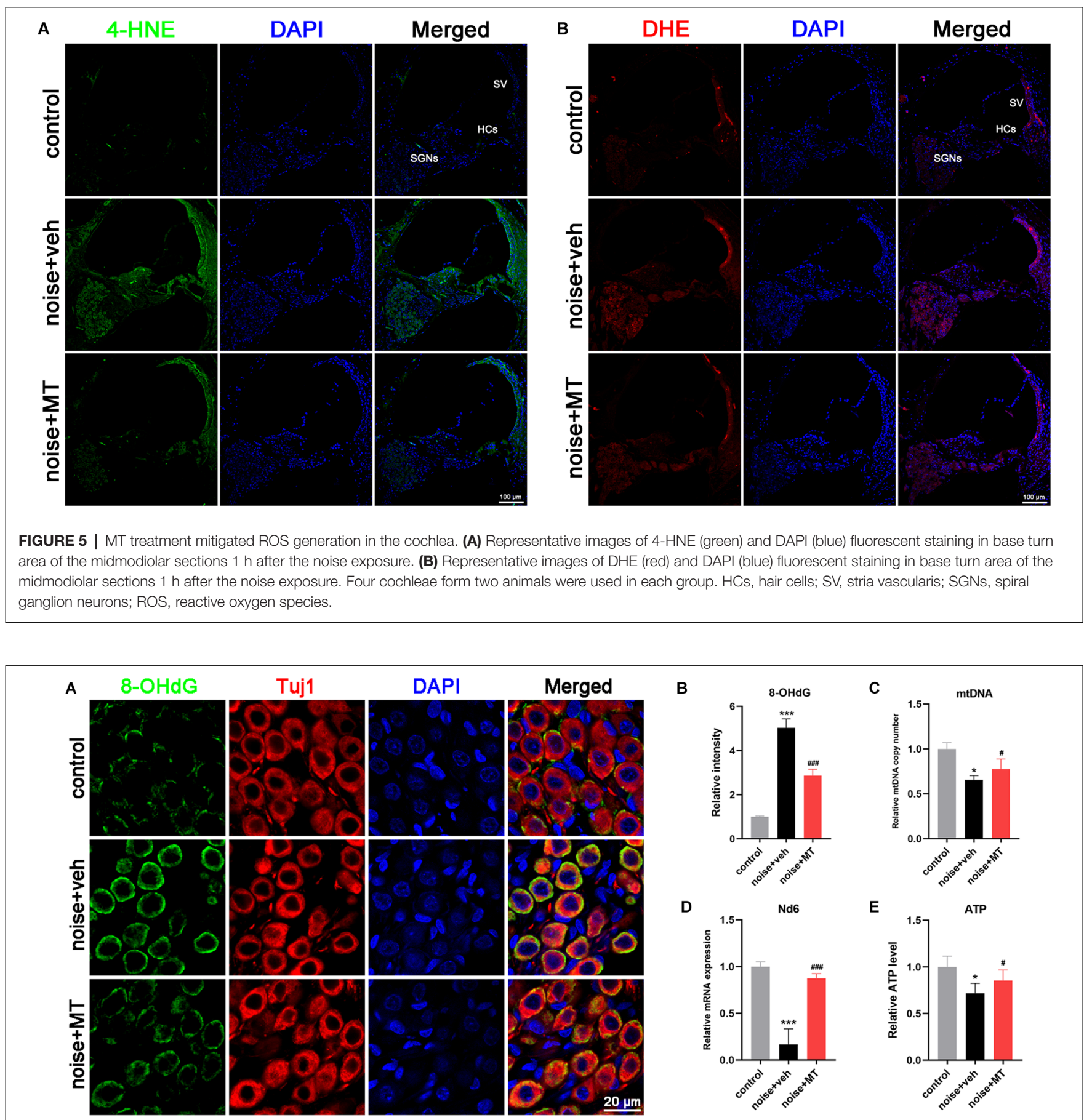

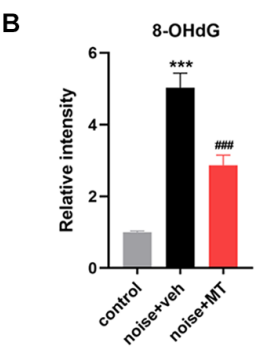

D

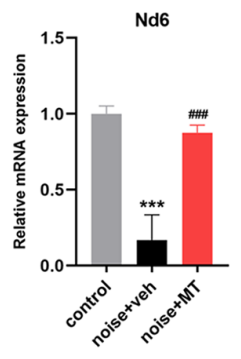

C

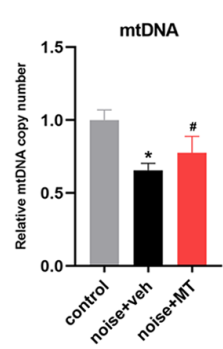

E

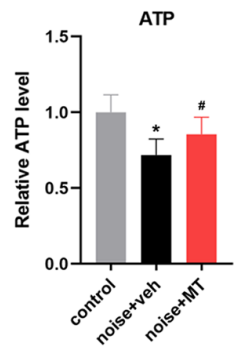

FIGURE 6 | MT treatment alleviated mtDNA oxidative damage, and maintained mitochondrial biogenesis and ATP production after the acoustic trauma. (A) Representative images of 8-OHdG (green), Tuj1 (red) and DAPI (blue) fluorescent staining in SGNs in frozen sections of the cochlea $1 \mathrm{~h}$ after the noise exposure. (B) Semi-quantitative analysis of the fluorescent intensity of 8-OHdG in SGNs $1 \mathrm{~h}$ after the noise exposure. (C) Real time-qPCR analysis of mtDNA copy number in the cochlea 3d after the noise exposure. (D) Real time-qPCR analysis of mitochondrial Nd6 expression level in the cochlear $1 \mathrm{~h}$ after the noise exposure. (E) The ATP level in the cochlea $1 \mathrm{~h}$ after the noise exposure. Data are presented as means $\pm \mathrm{SD}, n=4$ for each group, ${ }^{\star} p<0.05$ vs. control group, ${ }^{\star \star \star} p<0.001$ vs. control group, ${ }^{\#} p<0.05$ vs. vehicle-treated group, ${ }^{\# \# \# ~} p<0.001$ vs. vehicle-treated group.

(NIHHL; Kujawa and Liberman, 2009; Liberman and Kujawa, 2017). The protective effect of MT on ribbon synapses and ANFs suggested its promising application to prevent NIHHL, which needs further investigations.
The present study showed increased oxidative stress indicated by cochlear 4-HNE and DHE level, which is consistent with previous studies (Park et al., 2014; Paciello et al., 2020; He et al., 2021b). We observed increased mtDNA oxidative damage 

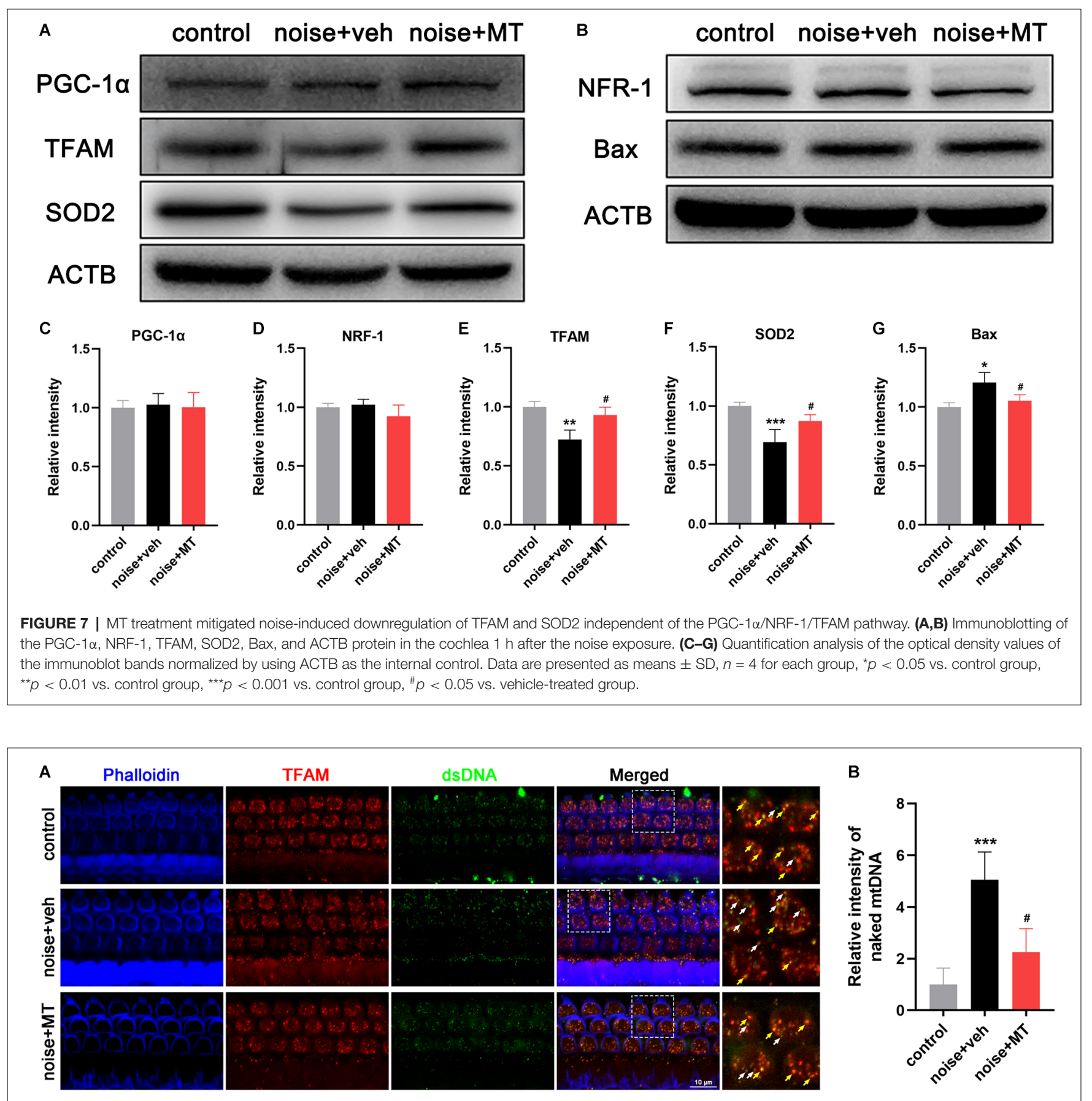

FIGURE 8 | MT treatment improved the disruption of TFAM-mtDNA interaction in the cochlea after the noise exposure. (A) Representative images of Phalloidin (blue), TFAM (red), and dsDNA (green) fluorescent staining in base turn area of the cochlear surface preparation $1 \mathrm{~h}$ after the noise exposure. Colocalized spots of TFAM and dsDNA were indicated in yellow arrows, while "orphan" dsDNA spots without colocalization of TFAM were indicated in the white arrows. (B) Quantification analysis of naked mtDNA in OHCs $1 \mathrm{~h}$ after the noise exposure. Data are presented as means $\pm \mathrm{SD}, n=4$ for each group, ${ }^{\star \star \star} p<0.001 \mathrm{vs}$. control group,

${ }^{\#} p<0.05$ vs. vehicle-treated group.

indicated by 8 -OHdG in SGNs $1 \mathrm{~h}$ after noise exposure. The cochlear mitochondrial function indicated by the ATP content was also impaired $1 \mathrm{~h}$ after the noise exposure, which is consistent with a previous study (Chen et al., 2012). Maintaining an adequate content of mtDNA copy number is important for cellular energy metabolism. Mitochondrial ROS induced by noise caused oxidative damage to mtDNA, resulting in mitochondrial dysfunction and energy insufficiency. SGNs are auditory neurons and consume a lot of energy in axoplasmic transport and electrical signal transmission. When the number 
and function of the mitochondria in SGNs are impaired, the transport of cellular cargos including neurotrophin might be disrupted, resulting in the degeneration of the neurites of SGNs. Mitochondrial biogenesis is an activity to generate new mitochondria from the existing ones. This process requires the coordination of mtDNA replication and expression and is regulated by several transcription factors. The present study showed that mtDNA content and expression level were both decreased after noise exposure, which indicated the impaired mitochondrial biogenesis in the inner ear after the acoustic trauma. Yu and colleagues found mtDNA common deletion induced by D-gal increased susceptibility to NIHL in rats (Yu et al., 2014), suggesting the correlation between mtDNA integrity and the pathogenesis of NIHL. Interestingly, we found that the mtDNA expression level decreased immediately following the acoustic trauma ( $1 \mathrm{~h}$ after the noise exposure). In contrast, mtDNA copy number did not reduce until the third day after the noise exposure. The possible explanation is that DNA is more stable and has a longer half-life than RNA. It takes time for mtDNA to be degraded after oxidative stress damage. Previous studies also showed that mtDNA copy numbers decrease several days after the environmental stress (Bagul et al., 2018; Sugasawa et al., 2021).

The PGC- $1 \alpha /$ NRF-1/TFAM axis is one of the most important pathways in regulating mitochondrial biogenesis. However, there are few studies on the alteration of the PGC- $1 \alpha / \mathrm{NRF}-1 / \mathrm{TFAM}$ pathway during acoustic trauma. In order to further explore the mechanism by which mtDNA content and expression are impaired in NIHL, we analyzed the expression level of this pathway. We found that the expression level of TFAM significantly decreased after the noise exposure, while the expression level of PGC- $1 \alpha$ and NRF-1 remained unchanged. In addition, the TFAM function, as indicated by the mtDNAbinding ability, was also disrupted in OHCs after the noise exposure. These results indicate that noise-induced ROS specifically impairs the expression and function of TFAM without affecting the upstream PGC-1 $\alpha$ and NRF-1. OHCs exhibit electromotility and mechanically amplify sound-evoked vibrations, which is an energy-consuming process. The impaired TFAM-mtDNA interaction leads to an increase of naked mtDNA molecules, which are vulnerable to oxidative damage by ROS. The abnormal mtDNA hinders the expression of mitochondrial respiratory chain proteins and ATP synthesis and might contribute to OHC dysfunction and death. A previous study showed that the phosphorylation and acetylation of TFAM within its HMG-box 1 domain reduced the DNA binding affinity, leading to TFAM-mtDNA disassembly (King et al., 2018). In addition, phosphorylation at serine $55 / 56$ facilitated rapid degradation of TFAM by the Lon protease in mitochondria ( $\mathrm{Lu}$ et al., 2013). Since it has been reported that acoustic trauma activates the protein kinase such as AMPK and extracellular signal-regulated protein kinase (ERK) in the inner ear (Kurioka et al., 2015; Hill et al., 2016), phosphorylation regulation might play a relevant role in the impaired expression and binding function of TFAM after the noise exposure. This speculation deserves further exploration in future studies.
Since oxidative stress plays an important role in the pathogenesis of NIHL, the antioxidants have been extensively studied in attenuating NIHL (Pak et al., 2020). A number of antioxidants show protective effects against NIHL in animal models such as glutathione, $\mathrm{N}$-acetylcysteine (NAC), Dmethionine, vitamin C, water-soluble coenzyme Q10, ebselen, resveratrol, and HK-2 (Ohinata et al., 2000; Seidman, 2003; Duan et al., 2004; Lynch et al., 2004; McFadden et al., 2005; Fetoni et al., 2009; Ewert et al., 2012; Chen et al., 2020), some of which have entered clinical trials (Kopke et al., 2015; Kil et al., 2017; Rosenhall et al., 2019). However, conventional antioxidants cannot remove only excess ROS without suppressing physiological ROS which are important for signal transduction. Recently, mitochondria-target antioxidants have been developed for the treatment of oxidative stressrelated disease for their capacity to scavenge ROS from the source (Fujimoto and Yamasoba, 2019). MT is the antioxidant TEMPOL conjugated to the lipophilic triphenylphosphonium (TPP) cation, which can easily pass through the phospholipid bilayer and accumulate several hundred-fold into mitochondria (Trnka et al., 2008). A previous study shows that MT is $\mathrm{BBB}$-penetrating and can be used as the contrast media in enhanced-magnetic resonance imaging (MRI) of the brain (Zhelev et al., 2013). In the present study, we demonstrated the existent of MT in the inner ear after the systemic administration. The lipophilic property of MT makes it easy to pass through the BLB and exert the antioxidant effects in the inner ear. MT exhibited a protective effect on hearing and cochlear cells against acoustic trauma. MT treatment reduced mitochondrial ROS production induced by noise exposure and therefore alleviated mtDNA oxidative damage and mitochondrial dysfunction. These results are consistent with a recent study (Zhao et al., 2021) which showed that MT alleviated ischemic acute kidney injury via reducing mitochondrial ROS and promoting TFAM-mediated mtDNA maintenance.

This study has several limitations. First, the methods used for hearing function evaluation were relatively simple. Four tested frequencies of the ABR covered about 50\% length of the cochlea (from $30 \%$ to $80 \%$ ), and may not represent well the hearing status of the animal. In future studies, we will use distortion product otoacoustic emissions (DPOAE) and compound action potential (CAP) for a comprehensive assessment of hearing function. Secondly, due to the technical difficulties, the RNA of HCs and SGNs was unable to be separately isolated. The alterations of mRNA expression of HCs and SGNs could not be analyzed respectively. Other methods such as RNA fluorescence in situ hybridization (FISH) will be used in our future studies. Finally, electron microscopy analysis of mitochondria was not performed in the present studies. Since mitochondrial fusion and fission are crucial processes to maintain mitochondrial homeostasis (Chan, 2020), the morphological changes of cochlear mitochondria in NIHL will be further studied in the following work.

In summary, our results first show TFAM reduction and the disruption of TFAM-mtDNA interaction in NIHL. Noiseinduced ROS lead to mtDNA oxidative damage, impairing mtDNA expression and mitochondrial biogenesis. MT treatment exhibits a protective effect on hearing, OHCs, 
IHC synapses, and ANFs against acoustic trauma partially by maintaining TFAM-mtDNA interaction and mitochondrial biogenesis based on its strong capacity of mitochondrial ROS scavenging.

\section{DATA AVAILABILITY STATEMENT}

The original contributions presented in the study are included in the article/Supplementary Material, further inquiries can be directed to the corresponding author/s.

\section{ETHICS STATEMENT}

The animal study was reviewed and approved by Institutional Animal Care and Use Committee of Fourth Military Medical University.

\section{AUTHOR CONTRIBUTIONS}

L-JL and QY designed the research. J-WC, P-WM, HY, W-LW, and P-HL performed the experiments. X-RD, Y-QL, and L-JL

\section{REFERENCES}

Abdullah-Al-Shoeb, M., Sasaki, K., Kikutani, S., Namba, N., Ueno, K., Kondo, Y., et al. (2020). The late-stage protective effect of mito-TEMPO against acetaminophen-induced hepatotoxicity in mouse and three-dimensional cell culture models. Antioxidants (Basel) 9:965. doi: 10.3390/antiox9100965

Bagul, P., Katare, P., Bugga, P., Dinda, A., and Banerjee, S. K. (2018). SIRT-3 modulation by resveratrol improves mitochondrial oxidative phosphorylation in diabetic heart through deacetylation of TFAM. Cells 7:235. doi: $10.3390 /$ cells7120235

Blazer, D. G., and Tucci, D. L. (2019). Hearing loss and psychiatric disorders: a review. Psychol. Med. 49, 891-897. doi: 10.1017/S0033291718003409

Böttger, E. C., and Schacht, J. (2013). The mitochondrion: a perpetrator of acquired hearing loss. Hear. Res. 303, 12-19. doi: 10.1016/j.heares.2013.01.006

Chan, D. C. (2020). Mitochondrial dynamics and its involvement in disease. Ann. Rev. Pathol. 15, 235-259. doi: 10.1146/annurev-pathmechdis-012419 $-032711$

Chandrasekaran, K., Anjaneyulu, M., Inoue, T., Choi, J., Sagi, A. R., Chen, C., et al. (2015). Mitochondrial transcription factor A regulation of mitochondrial degeneration in experimental diabetic neuropathy. Am. J. Physiol. Endocrinol. Metab. 309, E132-141. doi: 10.1152/ajpendo.00620.2014

Chen, G. D., Daszynski, D. M., Ding, D., Jiang, H., Woolman, T., Blessing, K., et al. (2020). Novel oral multifunctional antioxidant prevents noise-induced hearing loss and hair cell loss. Hear. Res. 388:107880. doi: 10.1016/j.heares.2019. 107880

Chen, F. Q., Zheng, H. W., Hill, K., and Sha, S. H. (2012). Traumatic noise activates Rho-family GTPases through transient cellular energy depletion. J. Neurosci. 32, 12421-12430. doi: 10.1523/JNEUROSCI.6381-11.2012

Coleman, J. K. M., Kopke, R. D., Liu, J., Ge, X., Harper, E. A., Jones, G. E., et al. (2007). Pharmacological rescue of noise induced hearing loss using $\mathrm{N}$-acetylcysteine and acetyl-1-carnitine. Hear. Res. 226, 104-113. doi: 10.1016/j. heares.2006.08.008

Di Domenico, F., Tramutola, A., and Butterfield, D. A. (2017). Role of 4-hydroxy2-nonenal (HNE) in the pathogenesis of Alzheimer disease and other selected age-related neurodegenerative disorders. Free Radic. Biol. Med. 111, 253-261. doi: 10.1016/j.freeradbiomed.2016.10.490

Ding, X., Wang, W., Chen, J., Zhao, Q., Lu, P., and Lu, L. (2019). Salidroside protects inner ear hair cells and spiral ganglion neurons from manganese exposure by regulating ROS levels and inhibiting apoptosis. Toxicol. Lett. 310, 51-60. doi: 10.1016/j.toxlet.2019.04.016 analyzed the data. J-WC prepared the figures. J-WC, QY, and L-JL wrote the manuscript. All authors contributed to the article and approved the submitted version.

\section{FUNDING}

This work was supported by the Military Logistics Research Project (18CXZ015).

\section{ACKNOWLEDGMENTS}

We thank Xi-Wang $\mathrm{Hu}$ and Ren-Feng Wang for their valuable guidance in image acquisition and $\mathrm{ABR}$ measurement. We also thank Qian Huo for proofreading the manuscript.

\section{SUPPLEMENTARY MATERIALS}

The Supplementary Material for this article can be found online at: https://www.frontiersin.org/articles/10.3389/fncel.2022.8037 18/full\#supplementary-material.

Du, K., Farhood, A., and Jaeschke, H. (2017). Mitochondria-targeted antioxidant mito-tempo protects against acetaminophen hepatotoxicity. Arch. Toxicol. 91 761-773. doi: 10.1007/s00204-016-1692-0

Duan, M., Qiu, J., Laurell, G., Olofsson, Å., Allen Counter, S., and Borg, E. (2004). Dose and time-dependent protection of the antioxidant $\mathrm{N}$-l-acetylcysteine against impulse noise trauma. Hear. Res. 192, 1-9. doi: 10.1016/j.heares.2004. 02.005

Ewert, D. L., Lu, J., Li, W., Du, X., Floyd, R., and Kopke, R. (2012). Antioxidant treatment reduces blast-induced cochlear damage and hearing loss. Hear. Res 285, 29-39. doi: 10.1016/j.heares.2012.01.013

Fernandez, K., Spielbauer, K. K., Rusheen, A., Wang, L., Baker, T. G., Eyles, S. et al. (2020). Lovastatin protects against cisplatin-induced hearing loss in mice. Hear. Res. 389:107905. doi: 10.1016/j.heares.2020.107905

Fetoni, A. R., Paciello, F., Rolesi, R., Eramo, S. L., Mancuso, C., Troiani, D., et al. (2015). Rosmarinic acid up-regulates the noise-activated Nrf2/HO-1 pathway and protects against noise-induced injury in rat cochlea. Free Radic. Biol. Med. 85, 269-281. doi: 10.1016/j.freeradbiomed.2015.04.021

Fetoni, A. R., Paciello, F., Rolesi, R., Paludetti, G., and Troiani, D. (2019). Targeting dysregulation of redox homeostasis in noise-induced hearing loss: oxidative stress and ROS signaling. Free Radic. Bio. Med. 135, 46-59. doi: 10.1016/j. freeradbiomed.2019.02.022

Fetoni, A. R., Piacentini, R., Fiorita, A., Paludetti, G., and Troiani, D. (2009). Water-soluble Coenzyme Q10 formulation (Q-ter) promotes outer hair cell survival in a guinea pig model of noise induced hearing loss (NIHL). Brain Res. 1257, 108-116. doi: 10.1016/j.brainres.2008.12.027

Fischel-Ghodsian, N., Kopke, R. D., and Ge, X. (2004). Mitochondrial dysfunction in hearing loss. Mitochondrion 4, 675-694. doi: 10.1016/j.mito.2004.07.040

Fujimoto, C., and Yamasoba, T. (2019). Mitochondria-targeted antioxidants for treatment of hearing loss: a systematic review. Antioxidants (Basel) 8:109. doi: 10.3390/antiox8040109

Gross, J., Olze, H., and Mazurek, B. (2014). Differential expression of transcription factors and inflammation-, ROS- and cell death-related genes in organotypic cultures in the modiolus, the organ of corti and the stria vascularis of newborn rats. Cell. Mol. Neurobiol. 34, 523-538. doi: 10.1007/s10571-014-0036-y

Han, S., Du, Z., Liu, K., and Gong, S. (2020). Nicotinamide riboside protects noiseinduced hearing loss by recovering the hair cell ribbon synapses. Neurosci. Lett. 725:134910. doi: 10.1016/j.neulet.2020.134910

He, Z., Guo, L., Shu, Y., Fang, Q., Zhou, H., Liu, Y., et al. (2017). Autophagy protects auditory hair cells against neomycin-induced damage. Autophagy 13, 1884-1904. doi: 10.1080/15548627.2017.1359449 
He, Z., Li, M., Fang, Q., Liao, F., Zou, S., Wu, X., et al. (2021a). FOXG1 promotes aging inner ear hair cell survival through activation of the autophagy pathway. Autophagy 17, 4341-4362. doi: 10.1080/15548627.2021. 1916194

He, Z., Pan, S., Zheng, H., Fang, Q., Hill, K., and Sha, S. (2021b). Treatment with calcineurin inhibitor FK506 attenuates noise-induced hearing loss. Front. Cell Dev. Biol. 9:648461. doi: 10.3389/fcell.2021.648461

Hill, K., Yuan, H., Wang, X., and Sha, S. H. (2016). Noise-induced loss of hair cells and cochlear synaptopathy are mediated by the activation of AMPK. J. Neurosci. 36, 7497-7510. doi: 10.1523/JNEUROSCI.0782 $-16.2016$

Kang, D., Kim, S. H., and Hamasaki, N. (2007). Mitochondrial transcription factor A (TFAM): Roles in maintenance of mtDNA and cellular functions. Mitochondrion 7, 39-44. doi: 10.1016/j.mito.2006.11.017

Kil, J., Lobarinas, E., Spankovich, C., Griffiths, S. K., Antonelli, P. J., Lynch, E. D., et al. (2017). Safety and efficacy of ebselen for the prevention of noise-induced hearing loss: a randomised, double-blind, placebo-controlled, phase 2 trial. Lancet 390, 969-979. doi: 10.1016/S0140-6736(17)31791-9

King, G. A., Hashemi, S. M., Taris, K. H., Pandey, A. K., Venkatesh, S., Thilagavathi, J., et al. (2018). Acetylation and phosphorylation of human TFAM regulate TFAM-DNA interactions via contrasting mechanisms. Nucleic Acids Res. 46, 3633-3642. doi: 10.1093/nar/gky204

Kopke, R., Slade, M. D., Jackson, R., Hammill, T., Fausti, S., Lonsbury-Martin, B., et al. (2015). Efficacy and safety of N-acetylcysteine in prevention of noise induced hearing loss: a randomized clinical trial. Hear. Res. 323, 40-50. doi: 10.1016/j.heares.2015.01.002

Kujawa, S. G., and Liberman, M. C. (2009). Adding insult to injury: cochlear nerve degeneration after "temporary" noise-induced hearing loss. J. Neurosci. 29, 14077-14085. doi: 10.1523/JNEUROSCI.2845-09.2009

Kurabi, A., Keithley, E. M., Housley, G. D., Ryan, A. F., and Wong, A. C. Y. (2017). Cellular mechanisms of noise-induced hearing loss. Hear. Res. 349, 129-137. doi: 10.1016/j.heares.2016.11.013

Kurioka, T., Matsunobu, T., Satoh, Y., Niwa, K., Endo, S., Fujioka, M., et al. (2015). ERK2 mediates inner hair cell survival and decreases susceptibility to noiseinduced hearing loss. Sci. Rep. 5:16839. doi: 10.1038/srep16839

Li, C., Sun, H., Xu, G., McCarter, K. D., Li, J., and Mayhan, W. G. (2018). Mito-tempo prevents nicotine-induced exacerbation of ischemic brain damage. J. Appl. Physiol. (1985) 125, 49-57. doi: 10.1152/japplphysiol.01084.2017

Liberman, M. C., and Kujawa, S. G. (2017). Cochlear synaptopathy in acquired sensorineural hearing loss: manifestations and mechanisms. Hear. Res. 349, 138-147. doi: 10.1016/j.heares.2017.01.003

Lin, M. T., and Beal, M. F. (2006). Mitochondrial dysfunction and oxidative stress in neurodegenerative diseases. Nature 443, 787-795. doi: 10.1038/nature05292

Liu, Y., Wang, Y., Ding, W., and Wang, Y. (2018). Mito-TEMPO alleviates renal fibrosis by reducing inflammation, mitochondrial dysfunction and endoplasmic reticulum stress. Oxid. Med. Cell. Longev. 2018:5828120. doi: $10.1155 / 2018 / 5828120$

Loughrey, D. G., Kelly, M. E., Kelley, G. A., Brennan, S., and Lawlor, B. A. (2018). Association of age-related hearing loss with cognitive function, cognitive impairment and dementia: a systematic review and meta-analysis. JAMA Otolaryngol. Head Neck Surg. 144, 115-126. doi: 10.1001/jamaoto. 2017.2513

Lu, B., Lee, J., Nie, X., Li, M., Morozov, Y. I., Venkatesh, S., et al. (2013). Phosphorylation of human TFAM in mitochondria impairs DNA binding and promotes degradation by the AAA+ lon protease. Mol. Cell 49, 121-132. doi: 10.1016/j.molcel.2012.10.023

Lynch, E. D., Gu, R., Pierce, C., and Kil, J. (2004). Ebselen-mediated protection from single and repeated noise exposure in rat. Laryngoscope 114, 333-337. doi: 10.1097/00005537-200402000-00029

McFadden, S. L., Woo, J. M., Michalak, N., and Ding, D. (2005). Dietary vitamin C supplementation reduces noise-induced hearing loss in guinea pigs. Hear. Res. 202, 200-208. doi: 10.1016/j.heares.2004.10.011

Nyberg, S., Abbott, N. J., Shi, X., Steyger, P. S., and Dabdoub, A. (2019). Delivery of therapeutics to the inner ear: the challenge of the blood-labyrinth barrier. Sci. Transl. Med. 11:eaao0935. doi: 10.1126/scitranslmed.aao0935

Ohinata, Y., Yamasoba, T., Schacht, J., and Miller, J. M. (2000). Glutathione limits noise-induced hearing loss. Hear. Res. 146, 28-34. doi: 10.1016/s03785955(00)00096-4
Ohlemiller, K. K., Wright, J. S., and Dugan, L. L. (1999). Early elevation of cochlear reactive oxygen species following noise exposure. Audiol. Neurootol. 4, 229-236. doi: 10.1159/000013846

Paciello, F., Di Pino, A., Rolesi, R., Troiani, D., Paludetti, G., Grassi, C., et al. (2020). Anti-oxidant and anti-inflammatory effects of caffeic acid: in vivo evidences in a model of noise-induced hearing loss. Food Chem. Toxicol. 143:111555. doi: 10.1016/j.fct.2020.111555

Pak, J. H., Kim, Y., Yi, J., and Chung, J. W. (2020). Antioxidant therapy against oxidative damage of the inner ear: protection and preconditioning. Antioxidants (Basel) 9:1076. doi: 10.3390/antiox 9111076

Park, J., Jou, I., and Park, S. M. (2014). Attenuation of noise-induced hearing loss using methylene blue. Cell Death Dis. 5:e1200. doi: 10.1038/cddis. 2014.170

Park, M., Lee, H. S., Song, J. J., Chang, S. O., and Oh, S. (2012). Increased activity of mitochondrial respiratory chain complex in noise-damaged rat cochlea. Acta Otolaryngol. 132, S134-S141. doi: 10.3109/00016489.2012. 659755

Rosenhall, U., Skoog, B., and Muhr, P. (2019). Treatment of military acoustic accidents with N-Acetyl-L-cysteine (NAC). Int. J. Audiol. 58, 151-157. doi: 10.1080/14992027.2018.1543961

Scarpulla, R. C. (2008). Transcriptional paradigms in mammalian mitochondrial biogenesis and function. Physiol. Rev. 88, 611-638. doi: 10.1152/physrev.00 025.2007

Seidman, M. (2003). Effects of resveratrol on acoustic trauma. Otolaryngol. Head Neck Surg. 129, 463-470. doi: 10.1016/s0194-5998(03)01586-9

Sha, S., and Schacht, J. (2017). Emerging therapeutic interventions against noise-induced hearing loss. Expert Opin. Investig. Drugs. 26, 85-96. doi: 10.1080/13543784.2017.1269171

Sha, S., Taylor, R., Forge, A., and Schacht, J. (2001). Differential vulnerability of basal and apical hair cells is based on intrinsic susceptibility to free radicals. Hear. Res. 155, 1-8. doi: 10.1016/s0378-5955(01)00224-6

Shetty, S., Kumar, R., and Bharati, S. (2019). Mito-TEMPO, a mitochondriatargeted antioxidant, prevents N-nitrosodiethylamine-induced hepatocarcinogenesis in mice. Free Radic. Bio. Med. 136, 76-86. doi: 10.1016/j. freeradbiomed.2019.03.037

Sugasawa, T., Ono, S., Yonamine, M., Fujita, S., Matsumoto, Y., Aoki, K., et al. (2021). One week of CDAHFD induces steatohepatitis and mitochondrial dysfunction with oxidative stress in liver. Int. J. Mol. Sci. 22:5851. doi: 10.3390/ijms22115851

Trnka, J., Blaikie, F. H., Smith, R. A. J., and Murphy, M. P. (2008). A mitochondria-targeted nitroxide is reduced to its hydroxylamine by ubiquinol in mitochondria. Free Radic. Bio. Med. 44, 1406-1419. doi: 10.1016/j. freeradbiomed.2007.12.036

Tsutsui, H., Kinugawa, S., and Matsushima, S. (2009). Mitochondrial oxidative stress and dysfunction in myocardial remodelling. Cardiovasc. Res. 81, 449-456. doi: 10.1093/cvr/cvn280

WHO. (2021). World report on hearing. Available online at: https://www.who.int/ publications/i/item/world-report-on-hearing.

Wu, F., Xiong, H., and Sha, S. (2020). Noise-induced loss of sensory hair cells is mediated by ROS/AMPK $\alpha$ pathway. Redox. Biol. 29:101406. doi: 10.1016/j. redox.2019.101406

Xing, H., Zhang, Z., Shi, G., He, Y., Song, Y., Liu, Y., et al. (2021). Chronic inhibition of mROS protects against coronary endothelial dysfunction in mice with diabetes. Front. Cell Dev. Biol. 9:643810. doi: 10.3389/fcell.2021. 643810

Xiong, H., Chen, S., Lai, L., Yang, H., Xu, Y., Pang, J., et al. (2019). Modulation of miR-34a/SIRT1 signaling protects cochlear hair cells against oxidative stress and delays age-related hearing loss through coordinated regulation of mitophagy and mitochondrial biogenesis. Neurobiol. Aging 79, 30-42. doi: 10.1016/j.neurobiolaging.2019.03.013

Yamashita, D., Jiang, H., Schacht, J., and Miller, J. M. (2004). Delayed production of free radicals following noise exposure. Brain Res. 1019, 201-209. doi: 10.1016/j.brainres.2004.05.104

Yu, J., Wang, Y., Liu, P., Li, Q., Sun, Y., and Kong, W. (2014). Mitochondrial DNA common deletion increases susceptibility to noise-induced hearing loss in a mimetic aging rat model. Biochem. Biophys. Res. Commun. 453, 515-520. doi: 10.1016/j.bbrc.2014.09.118 
Yuan, H., Wang, X., Hill, K., Chen, J., Lemasters, J., Yang, S. M., et al. (2015). Autophagy attenuates noise-induced hearing loss by reducing oxidative stress. Antioxid. Redox Signal. 22, 1308-1324. doi: 10.1089/ars.2014.6004

Zhao, X., Sun, J., Hu, Y., Yang, Y., Zhang, W., Hu, Y., et al. (2013). The effect of overexpression of PGC- $1 \alpha$ on the mtDNA4834 common deletion in a rat cochlear marginal cell senescence model. Hear. Res. 296, 13-24. doi: 10.1016/j. heares.2012.11.007

Zhao, M., Wang, Y., Li, L., Liu, S., Wang, C., Yuan, Y., et al. (2021). Mitochondrial ROS promote mitochondrial dysfunction and inflammation in ischemic acute kidney injury by disrupting TFAM-mediated mtDNA maintenance. Theranostics 11, 1845-1863. doi: 10.7150/thno.50905

Zhelev, Z., Bakalova, R., Aoki, I., Lazarova, D., and Saga, T. (2013). Imaging of superoxide generation in the dopaminergic area of the brain in Parkinson's disease, using Mito-TEMPO. ACS Chem. Neurosci. 4, 1439-1445. doi: $10.1021 / \mathrm{cn} 400159 \mathrm{~h}$

Zinovkin, R. A., and Zamyatnin, A. A. (2019). Mitochondria-targeted drugs. Curr. Mol. Pharmacol. 12, 202-214. doi: 10.2174/1874467212666181127151059
Conflict of Interest: The authors declare that the research was conducted in the absence of any commercial or financial relationships that could be construed as a potential conflict of interest.

Publisher's Note: All claims expressed in this article are solely those of the authors and do not necessarily represent those of their affiliated organizations, or those of the publisher, the editors and the reviewers. Any product that may be evaluated in this article, or claim that may be made by its manufacturer, is not guaranteed or endorsed by the publisher.

Copyright (C) 2022 Chen, Ma, Yuan, Wang, Lu, Ding, Lun, Yang and Lu. This is an open-access article distributed under the terms of the Creative Commons Attribution License (CC BY). The use distribution or reproduction in other forums is permitted provided the original author(s) the copyright owner(s) are credited that the original publication in this journal is cited in accordance with accepted academic practice. No use distribution or reproduction is permitted which does not comply with these terms. 